\title{
Systemic administration of bevacizumab prolongs survival in an in vivo model of platinum pre-treated ovarian cancer
}

\author{
DANIEL T. REIN ${ }^{1}$, ANNE KATHRIN VOLKMER ${ }^{1}$, JENS VOLKMER $^{2}$, INES M. BEYER $^{1,3}$, WOLFGANG JANNI ${ }^{1}$, \\ MARKUS C. FLEISCH ${ }^{1}$, ANNE KATHRIN WELTER ${ }^{1}$, DIRK BAUERSCHLAG ${ }^{4}$, \\ THOMAS SCHÖNDORF ${ }^{5}$ and MARTINA BREIDENBACH ${ }^{4}$
}

\begin{abstract}
Departments of ${ }^{1}$ Obstetrics and Gynaecology, and ${ }^{2}$ Urology, University of Düsseldorf Medical Centre, D-40225 Düsseldorf, Germany; ${ }^{3}$ Division of Medical Genetics, Department of Medicine, University of Washington, Seattle, WA 98195-7720, USA;

${ }^{4}$ Department of Obstetrics and Gynaecology, Rhine-Westphalian Technical University, D-52074 Aachen;

${ }^{5}$ Institute for Clinical Research and Development, D-55116 Mainz, Germany
\end{abstract}

Received October 17, 2011; Accepted December 21, 2011

DOI: $10.3892 / \mathrm{ol} .2012 .553$

\begin{abstract}
Ovarian cancer patients often suffer from malignant ascites and pleural effusion. Apart from worsening the outcome, this condition frequently impairs the quality of life in patients who are already distressed by ovarian cancer. This study investigated whether single intraperitoneal administration of the anti-VEGF antibody bevacizumab is capable of reducing the ascites-related body surface and prolonging survival. The study was performed in an orthotopic murine model of peritoneal disseminated platin-resistant ovarian cancer. Mice were treated with bevacizumab and/or paclitaxel or buffer (control). Reduction of body surface and increased survival rates were assessed as therapeutic success. Survival of mice in all treatment groups was significantly enhanced when compared to the non-treatment control group. The combination of paclitaxel plus bevacizumab significantly improved body surface as well as overall survival in comparison to a treatment with only one of the drugs. Treatment of malignant effusion with a single dose of bevacizumab as an intraperitoneal application, with or without cytostatic co-medication, may be a powerful alternative to systemic treatment.
\end{abstract}

\section{Introduction}

Despite advances in primary surgical therapy and first line chemotherapy, the majority of patients suffering from advanced surgical cancer ultimately relapse. Salvage therapy for platinum refractory disease or heavily pre-treated patients has proven unsuccessful with regard to survival. Therefore, therapy in

Correspondence to: Dr Daniel T. Rein, Department of Obstetrics and Gynaecology, University of Düsseldorf Medical Centre, Moorenstrasse 5, D-40225 Düsseldorf, Germany

E-mail: daniel.rein@hohenlind.de

Key words: advanced ovarian cancer, administration route, single doses, murine model, palliation these patients is primarily aimed at improving quality of life. Symptomatic malignant ascites remains a severe therapeutic challenge in patients suffering from recurrent ovarian cancer or other cancer types such as colorectal, stomach, pancreatic or lung cancer. Malignant ascites and pleural effusion are significant causes of morbidity and mortality, and frequently impair the quality of life in patients who are already distressed by the underlying disease. It is known that the median survival of patients suffering from malignant ascites is significantly reduced to 20 weeks following diagnosis if all cancer types are included $(1,2)$. However, effective treatment of malignant ascites in palliative patients is a therapeutic challenge and often frustrating. The established method of treating malignant ascites is paracentesis and the intraperitoneal (i.p.) instillation of chemotherapeutic agents such as cisplatin, 5-fluorouracil or doxorubicin $(3,4)$. However, the efficacy of i.p. chemotherapy in a palliative setting has only been investigated in small cohorts of highly selected patients. Although studies have shown partial responses, the results have been inconsistent and some studies have reported significant side-effects (5).

A better understanding of the underlying molecular mechanisms that regulate the formation of malignant effusions have led to the concept that the angiogenic biomolecule vascular epithelial growth factor (VEGF) is important in the pathobiology of a variety of tumours and the pathogenesis of malignant and non-malignant effusions (6). It promotes tumour angiogenesis, formation of ascites and pleural effusions, and metastasis in ovarian cancer (7). Inhibitors of VEGF, such as the antibody bevacizumab, suppress tumour growth in ovarian cancer models, possibly by inhibiting angiogenesis (7). It is known that in patients with malignant effusions, high levels of VEGF are found in serum/plasma as well as in the malignant effusion (8). Previous studies showed a positive therapeutic effect of bevacizumab in the systemic treatment of patients with ovarian carcinoma and formation of ascites (9). Numnum et al demonstrated that patients undergoing treatment with bevacizumab showed a symptomatic relief of ascites and required no therapeutic paracentesis after treatment with the antibody (9). The most common serious bevacizumab-related toxicities are 
hypertension, fatigue, proteinuria, bleeding and pain (10). Some cases of gastrointestinal perforation or fistula, brain ischemia, pulmonary hypertension, gastrointestinal bleeding and wound healing complications have been reported (11).

Intraperitoneal administration of bevacizumab has recently been demonstrated to be efficacious in locally pretreated cancer patients suffering from malignant ascites (12). Since malignant pleural effusion contains high numbers of active tumour cells, the local intrapleural application of bevacizumab is a rational alternative to intravenous (i.v.) administration. However, to date only a few patients with malignant ascites have been treated with intraperitoneally delivered bevacizumab, and therefore this route of administration needs to be further explored. In this study, we have compared i.v. and i.p. administration of bevacizumab in an ovarian cancer mouse model with intraperitoneal metastasis. We have examined whether a single i.p. administration of bevacizumab is capable of reducing ascites-related body surface and prolonging survival.

\section{Materials and methods}

Cells and cell lines. SKOV-3.ip1 ovarian adenocarcinoma cell lines were kind gifts from Drs Judy Wolf and Janet Price (University of Texas MD Anderson Cancer Center, Houston, TX, USA), respectively. Cell lines were maintained in recommended conditions. Cells were grown at $37^{\circ} \mathrm{C}$ in a humidified atmosphere of $5 \% \mathrm{CO}_{2}$.

To generate resistant ovarian cancer cells, SKOV-3.ip1 cells were cultured in growth medium (GM) containing cisplatin. Initially, $10 \mathrm{ng} / \mathrm{ml}$ cisplatin was added to the GM and the dose was increased by $10 \mathrm{ng} / \mathrm{ml}$ per week to a final concentration of $60 \mathrm{ng} / \mathrm{ml}$.

Anticancer drugs. Paclitaxel and novantrone were obtained from the pharmacy of the University of Düsseldorf Medical Centre and handled according to the manufacturer's instructions. Paclitaxel $(6 \mathrm{mg} / \mathrm{ml})$ was diluted at a ratio of $1: 15$ with saline. Novantrone $(2 \mathrm{mg} / \mathrm{ml})$ was diluted at a ratio of $1: 50$ with saline. Bevacizumab was purchased from Roche (Basel, Switzerland). Each mouse assigned to a bevacizumab treatment received a single application. Bevacizumab was diluted in $200 \mu \mathrm{l}$ of phosphate-buffered saline (PBS) immediately prior to administration.

Therapeutic ovarian cancer model. Female CB17 SCID mice were obtained at 3-4 weeks of age and quarantined at least 1 week prior to the study. Mice were kept under pathogen-free conditions according to the American Association for Accreditation of Laboratory Animal Care guidelines. Animal protocols were reviewed and approved by the state authorities according to the German animal protection law. Mice were injected i.p. with $1 \times 10^{7}$ drug resistant SKOV-3.ip1 cells on day 0 . Mice were assigned into six treatment groups, each consisting of 11 mice: Group 1 served as the control (injection of PBS); Group 2 and 3 received bevacizumab i.p. or i.v., respectively; Group 4 was treated with paclitaxel; the therapy of Group 5 and 6 consisted of paclitaxel plus bevacizumab i.p. or i.v., respectively. Each mouse assigned to a chemotherapy treatment received 2 applications of paclitaxel $(10 \mathrm{mg}$ per $\mathrm{kg}$ body weight) on days 11 and 13 . Bevacizumab was adminis- tered at a total dose of $10 \mathrm{mg}$ per kg body weight on day 12 . Drug injections were performed either intravenously through the lateral tail vein (i.v. group) or via the peritoneum (i.p. group).

Abdominal circumference and body weight were measured weekly for calculation of the body surface until 35 days following initial treatment. Variations in body surface are dependent on ascites volume plus tumour burden and therefore the body surface is considered a valuable parameter for disease progression.

A total of 21 days following the initial treatment, a representative mouse from each treatment group was sacrificed and the expression of VEGF was examined in the macroscopic disease and in the ascites. In the remaining mice, survival was followed daily until mortality.

$R N A$ preparation and quantitative $R T-P C R$. The total cellular RNA of tumour cells was extracted from $2 \times 10^{5}$ cells using the RNeasy mini prep kit (Qiagen, Santa Clarita, CA, USA) and additionally treated with DNase I (Life Technologies Inc; Rockville, MD, USA) for $30 \mathrm{~min}$. PCR products from the VEGF gene were used for the creation of the standard curve. A GeneAmp RNA PCR core kit (Applied Biosystems, Foster City, CA, USA) was used for cDNA synthesis and PCR amplification of cDNA products. TaqMan primers and probes were designed by the Primer Express 1.0 software and synthesized by Applied Biosystems. Oligonucleotide sequences for the amplification of the VEGF gene were: forward primer CAT GCA GAT TAT GCG GAT CAA; reverse primer TTT GTT GTG CTG TAG GAA GCT CA; and probe 6FAM- CCT CAC CAA GGC CAG CAC ATA GGA GA-TAMRA. The human housekeeping gene glyceraldehydes-3-phosphate dehydrogenase (GAPDH) served as an internal control.

Each single real-time PCR contained 1X TaqMan ${ }^{\circledR}$ EZ RT-PCT kit (Applied Biosystems), $100 \mathrm{nM}$ forward primer, $100 \mathrm{nM}$ reverse primer, $100 \mathrm{nM}$ probe and $0.025 \%$ bovine serum albumin in a final volume of $9 \mu \mathrm{l}$ per reaction. The PCR contained $1 \mu \mathrm{l}$ of template or water as the control.

PCR was carried out using a LightCyclerTMSystem (Roche Molecular Biochemicals, Indianapolis, IN, USA) according to the manufacturer's instructions. Thermal cycling conditions were: $2 \mathrm{~min}$ at $50^{\circ} \mathrm{C}, 30 \mathrm{~min}$ at $60^{\circ} \mathrm{C}, 5 \mathrm{~min}$ at $95^{\circ} \mathrm{C}$ and 40 cycles of $20 \mathrm{sec}$ at $94^{\circ} \mathrm{C}$ and $1 \mathrm{~min}$ at $60^{\circ} \mathrm{C}$. Data were analyzed with LightCycler software. Known amounts of human total RNA $(200,20,2$ and $0.2 \mathrm{ng} / \mu \mathrm{l})$ were amplified to generate a standard curve for the determination of the concentration of the unknown samples.

Statistical analysis. Data were presented as the mean values \pm standard deviation. The body surface among groups was assessed with a two-tailed Student's t-test. $\mathrm{P}<0.05$ was considered to indicate a statistically significant difference. Survival data were plotted as a Kaplan-Meier curve. Statistical analyses were performed using Prism 4 software (GraphPad Software, San Diego, CA, USA).

\section{Results}

The orthotopic murine model of peritoneal disseminated ovarian cancer. One representative mouse per treatment group 


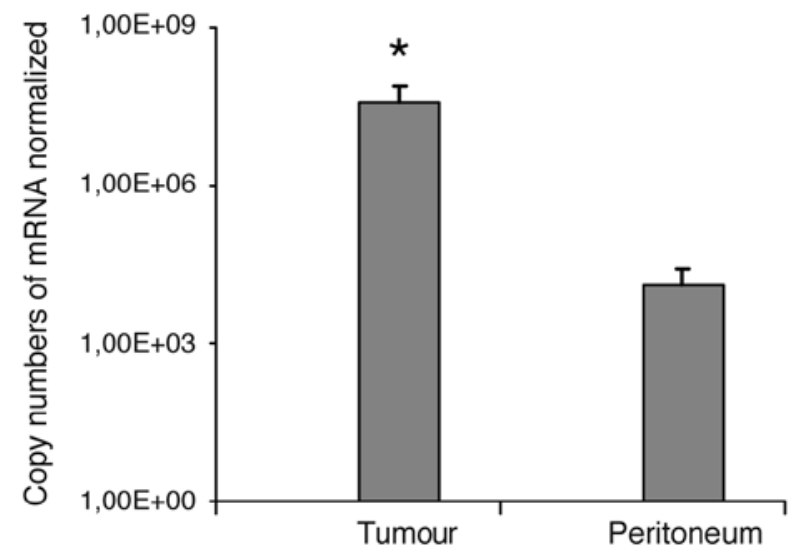

Figure 1. VEGF expression in ovarian cancer tissue compared to healthy peritoneum. The data confirm that anti-VEGF therapy is likely to address ovarian tumours. Each bar is the mean of 6 mice $\pm \mathrm{SD}$. ${ }^{*} \mathrm{P}<0.05$. VEGF, vascular epithelial growth factor.

was investigated to confirm the tumour model. The dissemination of ovarian cancer was confirmed surgically. Samples of each tumour burden were successfully checked for VEGF overexpression. Fig. 1 shows the data of all six sacrificed mice. VEGF was expressed 2841-fold $(\mathrm{p}<0.001)$ higher in the tumour tissues than in the peritoneum, which was defined as the healthy control tissue.

Overall survival. Advanced carcinomatosis was allowed to develop for 12 days and mice were treated with either a single dose of PBS (negative control), bevacizumab i.v., bevacizumab i.p., paclitaxel i.p. or a combination of bevacizumab and paclitaxel. Fig. 2 shows the results in a plot according to Kaplan-Meier calculations. In pairwise comparisons, survival of mice in all treatment groups was significantly enhanced when compared to the non-treatment control group $(p<0.001$ for bevacizumab + paclitaxel and $p<0.05$ for single agent bevacizumab i.p., bevacizumab i.v. and paclitaxel i.p.). Survival of mice treated with a combination regimen of bevacizumab and paclitaxel was significantly prolonged compared to mice treated with a single agent $(\mathrm{p}<0.001)$. However, differences between i.p. and i.v. administration of bevacizumab were not statistically significant in the single agent regimen or combination regimen with paclitaxel.

Therapeutic success. Success of the treatment was assessed via reduction of the body surface, which is proportional to the intracorporal ascites volume.

As shown in Fig. 3, the mean abdominal surface (AS) 24 days after initiation of treatment was 376, 266, 298, 272, 231 and $234 \mathrm{~mm}^{2}$ for mice treated with PBS, bevacizumab i.p., bevacizumab i.v., paclitaxel i.p, bevacizumab i.p. + paclitaxel i.p. or bevacizumab i.v. + paclitaxel i.p., respectively. The mean AS of all mice prior to treatment was $227 \pm 22 \mathrm{~mm}^{2}$. The results of the statistical analyses among the treatment arms are shown in Table I. Each treatment resulted in a significant decrease of body surface with the exception of bevacizumab i.v. In that group, two mice were non-responders to the therapy and showed a body surface comparable to the animals without treatment. It is noteworthy that the combination of paclitaxel plus bevacizumab
Table I. Summary of the statistical analyses shown as the p-values following body surface comparisons using the t-test.

P-value

vs. no treatment

Bevacizumab i.p.

0.00113

Bevacizumab i.v.

0.7159

Paclitaxel i.p.

0.00279

Paclitaxel i.p. + bevacizumab i.p.

0.000148

Paclitaxel i.p. + bevacizumab i.v.

0.0000065

vs. paclitaxel or bevacizumab

Paclitaxel + bevacizumab

0.00000577

All treatment arms, except one group (bevacizumab i.v.), showed statistically significant improvement.
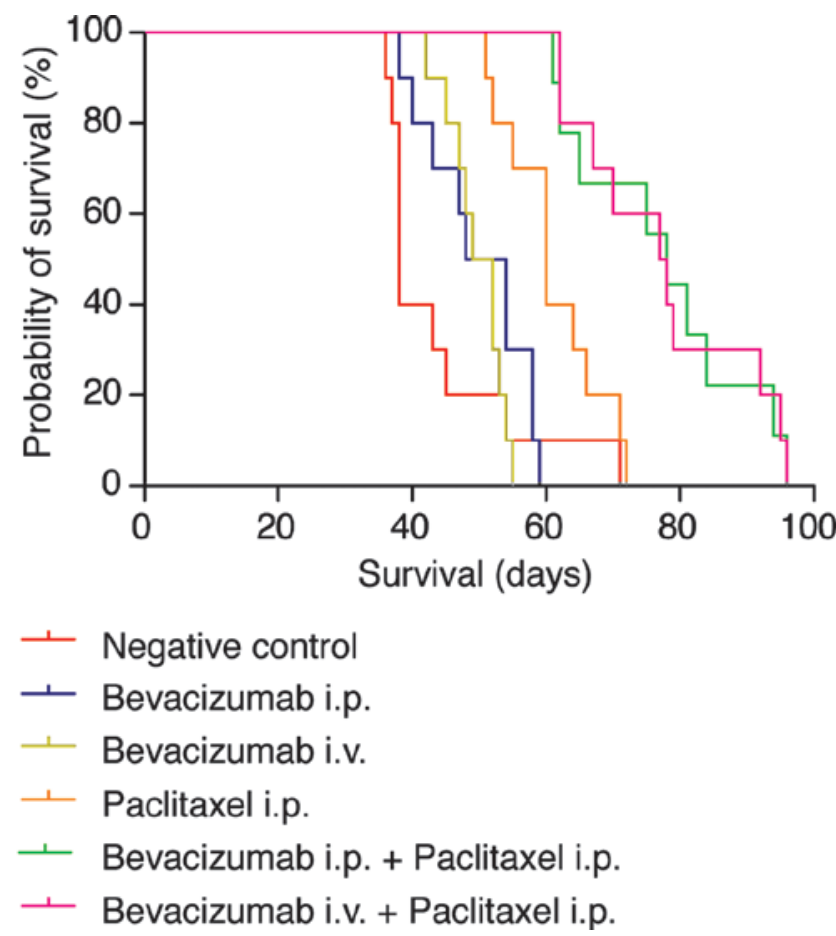

Figure 2. Overall survival of the six treatment groups, shown according to Kaplan-Meier curves. The therapeutic effect of bevacizumab \pm paclitaxel was evaluated in an animal model of peritoneally disseminated ovarian cancer. SKOV-3.ip1 cells were injected intraperitoneally (i.p.) into SCID mice and advanced carcinomatosis was allowed to develop for 12 days. On day 13 mice were injected with bevacizumab i.p., bevacizumab intravenously (i.v.), paclitaxel i.p., bevacizumab (i.p. or i.v.) + paclitaxel or PBS only (negative control). Administration of the combination regimen resulted in significantly enhanced survival. SCID, severe combined immunodeficiency; PBS, phosphate-buffered saline.

significantly improved body surface as well as overall survival in comparison to a treatment with one of the drugs.

\section{Discussion}

Ovarian carcinoma is the leading cause of mortality from gynaecological cancer (7). In general, the tumour progression 
A

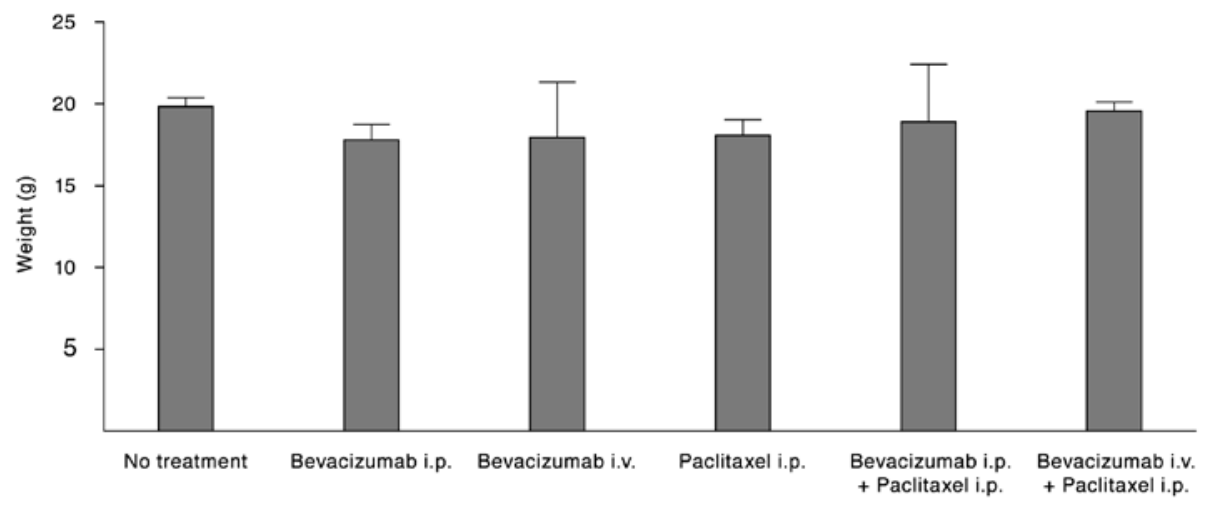

B

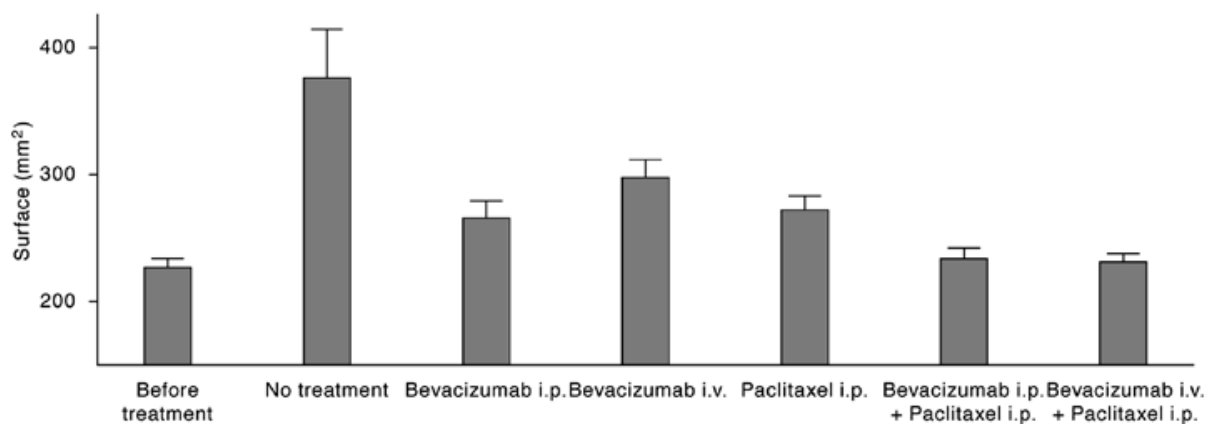

Figure 3. Effect of the respective treatment (bevacizumab \pm paclitaxelor control) on (A) the body weight and (B) surface of mice. Each bar represents the mean of 11 mice \pm SD.

is already advanced at the time of diagnosis. Despite advances in primary surgical therapy and first line chemotherapy, the majority of patients suffering from advanced surgical cancer ultimately relapse. Salvage therapy for platinum refractory disease has shown limited efficacy with regard to survival. Therapy in these patients is therefore primarily aimed at improving quality of life. Secondary symptoms such as symptomatic malignant ascites are a therapeutic challenge in the palliative setting. In addition, it is well known that the prognosis of patients with malignant pleural effusion is worse compared to those without secondary symptoms (1). The effect of paracentesis is usually of short duration and the effect of i.p. administration of chemotherapeutic agents is limited.

The humanized monoclonal antibody bevacizumab that targets angiogenesis by binding to VEGF has shown significant single agent activity in patients suffering from recurrent ovarian cancer. Studies have shown response rates between 16 and $18 \%$ in platinum-sensitive and platinum-refractory disease $(10,13)$. Despite the improvement of progression-free survival in these trials, bevacizumab appears to improve the control of ascites. These data are in line with the observation of elevated levels of VEGF in malignant ascites $(6,14)$.

Evidence suggests that angiogenesis is important in the prognosis and progression of epithelial ovarian cancer. Several studies have demonstrated a direct association of microvessel density and VEGF in primary tumours with extent of disease and time to progression (15). In addition, VEGF has relatively low levels of expression in normal tissue (16) and is therefore considered attractive as a potential target for an anti-VEGF agent such as bevacizumab. Preclinical models have demonstrated inhibition of tumour growth in various tumour cell types (17). Several alterations in vascular function, such as decreases in vessel density, diameter and permeability, result in a decreased production of malignant ascites. Consequently, various approaches have been examined to target VEGF and VEGF receptors (18), with several studies having demonstrated a reduction of ascites and tumour growth inhibition following the administration of bevacizumab in various ovarian cancer animal models (19).

Findings of a recently published study have shown prolonged survival following maintenance therapy in an intraperitoneal xenograft mouse model of previously untreated ovarian cancer (20). This study showed a significant inhibition of tumour growth following administration of $5 \mathrm{mg} / \mathrm{kg}$ bevacizumab as a single agent. In addition, bevacizumab significantly enhanced sensitivity to cisplatin. It may be speculated that enhancement of drug-induced tumour cell apoptosis by VEGF immunoneutralisation is the molecular basis which has been shown recently for cisplatin-treated ovarian cancer (21).

Another study has recently demonstrated rapid absorption of bevacizumab following i.p. administration, with a bioavailability of $92.8 \%$ (23). The authors reported a rapid and near complete absorption of bevacizumab following i.p. dosing, as well as increased animal survival following combined therapy with bevacizumab and i.p. bevacizumab and i.p. paclitaxel. As yet, little is known about the effect of bevacizumab on malignant ascites in pre-treated ovarian cancer patients. To the best of our knowledge, the present study is the first that evaluated i.v. as well as i.p. administration of bevacizumab in a xenograft model of cisplatin pre-treated ovarian cancer. Of note, we only administered a single dose of bevacizumab, paclitaxel or the combination therapy. We demonstrated significantly 
prolonged survival and reduction of ascites. Moreover, the addition of bevacizumab to a common cytostatic therapy such as paclitaxel resulted in further improvement in ascites reduction and survival. These findings are in agreement with preclinical and clinical studies which demonstrated that the efficacy of i.p. chemotherapy is limited by poor penetration of drugs into peritoneal tumours (23). It has been hypothesized, that adjunct therapy with anti-angiogenic agents may lead to decreased drug removal from peritoneal tumours, increased drug concentration in tumours and increased efficacy of i.p. chemotherapy (21). In a recently published report, four heavily pre-treated ovarian cancer patients received bevacizumab intravenously at a dose of $15 \mathrm{mg} / \mathrm{kg}$ every 3 weeks $(9,17)$. Ascites were significantly reduced in all patients and no patient required a paracentesis following the initiation of therapy. Only a few patients with malignant ascites have received i.p. bevacizumab (4). Nine patients with malignant ascites from colon, breast, uterine and ovarian cancer, for whom repeated paracentesis prior to treatment was required, were administered intraperitoneal bevacizumab at $5 \mathrm{mg} / \mathrm{kg}$ monthly. Eight out of nine patients $(89 \%)$ experienced prolongation of the interval between paracentesis (23). However, the i.p. route of administration in a palliative patient population needs to be examined stringently regarding the safety of the treatment. Intraperitoneal application may be the route of choice in this particular setting as it allows for the accumulation of the study drug within the body compartment where malignant ascites is promoted by VEGF secretion.

The effects of bevacizumab, either alone or in combination with i.p. paclitaxel, were not found to be significantly dependent on the route of administration. We conclude from this study that treatment of malignant ascites with i.p. bevacizumab is an effective and powerful alternative to i.v. treatment.

\section{References}

1. Eitan R, Levine DA, Abu-Rustum N, et al: The clinical significance of malignant pleural effusions in patients with optimally debulked ovarian carcinoma. Cancer 103: 1397-1401, 2005.

2. Sherer DM, Eliakim R and Abulafia O: The role of angiogenesis in the accumulation of peritoneal fluid in benign conditions and the development of malignant ascites in the female. Gynecol Obstet Invest 50: 217-224, 2000.

3. Markman M: Intraperitoneal antineoplastic drug delivery: rationale and results. Lancet Oncol 4: 277-283, 2003.

4. Kobold S, Hegewisch-Becker S, Oechsle K, Jordan K, Bokemeyer C and Atanackovic D: Intraperitoneal VEGF inhibition using bevacizumab: a potential approach for the symptomatic treatment of malignant ascites? Oncologist 14: 1242-1251, 2009.

5. Aslam N and Marino CR: Malignant ascites: new concepts in pathophysiology, diagnosis, and management. Arch Intern Med 161: 2733-2737, 2001.
6. Senger DR, Galli SJ, Dvorak AM, Perruzzi CA, Harvey VS and Dvorak HF: Tumor cells secrete a vascular permeability factor that promotes accumulation of ascites fluid. Science 219: 983-985, 1983.

7. Huynh H, Teo CC and Soo KC: Bevacizumab and rapamycin inhibit tumor growth in peritoneal model of human ovarian cancer. Mol Cancer Ther 6: 2959-2966, 2007.

8. Sack U, Hoffmann M, Zhao XJ, et al: Vascular endothelial growth factor in pleural effusions of different origin. Eur Respir J 25: 600-604, 2005 .

9. Numnum TM, Rocconi RP, Whitworth J and Barnes MN: The use of bevacizumab to palliate symptomatic ascites in patients with refractory ovarian carcinoma. Gynecol Oncol 102: 425-428, 2006.

10. Cannistra SA, Matulonis UA, Penson RT, et al: Phase II study of bevacizumab in patients with platinum-resistant ovarian cancer or peritoneal serous cancer. J Clin Oncol 25: 5180-5186, 2007.

11. Simpkins F, Belinson JL and Rose PG: Avoiding bevacizumab related gastrointestinal toxicity for recurrent ovarian cancer by careful patient screening. Gynecol Oncol 107: 118-123, 2007.

12. Hamilton CA, Maxwell GL, Chernofsky MR, Bernstein SA, Farley JH and Rose GS: Intraperitoneal bevacizumab for the palliation of malignant ascites in refractory ovarian cancer. Gynecol Oncol 111: 530-532, 2008.

13. Burger RA, Sill MW, Monk BJ, Greer BE and Sorosky JI: Phase II trial of bevacizumab in persistent or recurrent epithelial ovarian cancer or primary peritoneal cancer: a Gynecologic Oncology Group Study. J Clin Oncol 25: 5165-5171, 2007.

14. Zebrowski BK, Liu W, Ramirez K, Akagi Y, Mills GB and Ellis LM: Markedly elevated levels of vascular endothelial growth factor in malignant ascites. Ann Surg Oncol 6: 373-378, 1999.

15. Ferrara N: Vascular endothelial growth factor: basic science and clinical progress. Endocr Rev 25: 581-611, 2004.

16. Rein DT, Breidenbach M, Nettelbeck DM, et al: Evaluation of tissue-specific promoters in carcinomas of the cervix uteri. J Gene Med 6: 1281-1289, 2004.

17. Gerber HP and Ferrara N: Pharmacology and pharmacodynamics of bevacizumab as monotherapy or in combination with cytotoxic therapy in preclinical studies. Cancer Res 65: 671-680, 2005.

18. Jain RK, Duda DG, Clark JW and Loeffler JS: Lessons from phase III clinical trials on anti-VEGF therapy for cancer. Nat Clin Pract Oncol 3: 24-40, 2006.

19. Hu L, Hofmann J, Zaloudek C, Ferrara N, Hamilton T and Jaffe RB: Vascular endothelial growth factor immunoneutralization plus Paclitaxel markedly reduces tumor burden and ascites in athymic mouse model of ovarian cancer. Am J Pathol 161: 1917-1924, 2002.

20. Mabuchi S, Terai Y, Morishige K, et al: Maintenance treatment with bevacizumab prolongs survival in an in vivo ovarian cancer model. Clin Cancer Res 14: 7781-7789, 2008.

21. Shah DK, Shin BS, Veith J, Toth K, Bernacki RJ and Balthasar JP. Use of an anti-vascular endothelial growth factor antibody in a pharmacokinetic strategy to increase the efficacy of intraperitoneal chemotherapy. J Pharmacol Exp Ther 329: 580-591, 2009.

22. Davies S, Dai D, Pickett G, Thiel KW, Korovkina VP and Leslie KK: Effects of bevacizumab in mouse model of endometrial cancer: Defining the molecular basis for resistance. Oncol Rep 25: 855-862.

23. Shah DK, Veith J, Bernacki RJ and Balthasar JP: Evaluation of combined bevacizumab and intraperitoneal carboplatin or paclitaxel therapy in a mouse model of ovarian cancer. Cancer Chemother Pharmacol 68: 951-958, 2011. 